\title{
Desarrollo de la expresión oral y de la oralidad a través de los clubs de lectura en el aula de inglés en Escuelas Oficiales de Idiomas
}

a

b

Sonia Vecino-Ramos y Paola Ruiz-Bernardo

a

Universidad Jaume I (Castellón, al400470@uji.es), Universidad Jaume I (Castellón, ruizma@uji.es).

\section{Abstract}

The reading clubs or book clubs are an innovative practice in the foreign language classroom at Official Schools of Languages that allow the students to share their individual and personal experience with their classmates who have read the same book. In this way they improve their speaking by means of the practice of orality and, at the same time, their critical and reflexive thinking are promoted.

This communication explains the case study of these reading clubs in English classes at Official School of Languages in Castellón in the B1, B2 and C1 levels.

To evaluate the experience a self-designed survey was administered based on the students' reading development and centred in their perception related to their improvement in reading comprehension and oral expression.

From the results it can be concluded that the students perception towards the use of reading clubs in the classroom to practise orality and speaking is positive, which makes it advisable to use them in other languages and different educational levels.

Keywords: educational innovation, book clubs, oral expression, Official Schools of Languages, orality, critical thinking

\section{Resumen}

Los clubs o círculos de lectura son una práctica innovadora en el aula de lenguas extranjeras de Escuelas Oficiales de Idiomas que permiten que el alumnado comparta su experiencia individual y personal con el resto de compañeros que han leído la misma obra. De esta manera mejoran su expresión oral a través de la práctica de la oralidad y, al mismo tiempo, se promueve el pensamiento crítico y reflexivo.

La presente comunicación relata el estudio de caso de estos clubs de lectura en clases de inglés de la Escuela Oficial de Idiomas de Castellón en los niveles B1, B2 y C1.

Para evaluar la experiencia se administró un cuestionario de elaboración propia basado en el desarrollo lector de los alumnos y centrado en su percepción con respecto a la mejora de su comprensión lectora y de su expresión oral.

De los resultados se concluye que la percepción de los estudiantes es positiva, por lo que se recomienda su uso en otros idiomas y diferentes niveles educativos.

Palabras clave: Innovación educativa, clubs de lectura, expresión oral, Escuelas Oficiales de Idiomas, oralidad, pensamiento crítico 


\section{Introducción}

El uso de los libros de lectura en el aprendizaje de la lengua, tanto materna como extranjera, es de uso muy extendido y tiene una larga tradición en la docencia. Esta práctica por lo general siempre se ha hecho desde la vertiente del desarrollo de la comprensión lectora, realizando la lectura de manera individual y después un examen o prueba práctica, como pueden ser presentaciones orales.

Según Koutsompou (2015) el uso de la literatura en la enseñanza de la lengua ayuda al alumno a trabajar con ejemplos reales y le expone a una variedad de registros y a saber diferenciar el propósito de cada uno de ellos. Por otro lado, citando a la misma autora, el texto literario tiene múltiples interpretaciones y genera diferentes opiniones entre los alumnos, lo que deriva en una interacción real y motivadora con el texto y con los alumnos (p. 75). Este último punto se ve también favorecido por la aplicación del aprendizaje dialógico mediante el contraste de ideas (Moliner, Sanahuja y Benet, 2017) que se da en los grupos del club de lectura basados en roles cooperativos que proponemos en la experiencia didáctica que presentamos en esta comunicación.

Dada la importancia de la exposición a la lengua en versión original y a los beneficios de la lectura en general, la actual propuesta de innovación educativa surge hace ya más de diez años a partir de la idea de los clubs de lectura que realizan bibliotecas, librerías, asociaciones y centros culturales y poco a poco se empezó a implementar en las aulas de las Escuelas Oficiales de Idiomas. Tal como expone Álvarez (2016): "Las prácticas de lectura más relevantes en la actualidad se enmarcan en el paradigma de la lectura dialógica, dando relevancia no solo a la lectura sino también a la interacción entre lectores. Una de estas prácticas son los clubs de lectura, que en España se vienen desarrollando desde hace treinta y cinco años: en bibliotecas públicas, librerías, centros cívicos, etc" (p. 7).

A partir de la utilización continuada de los clubs de lectura en el aula de inglés se ha observado que los estudiantes no son conscientes de la importancia que tiene la expresión oral en esta actividad, puesto que parece que todo se centra en la lectura de la obra literaria, cuando uno de los objetivos importantes junto con el desarrollo de la comprensión lectora es la práctica de la oralidad.

Las ventajas de utilizar los clubs de lectura con respecto a las actividades tradicionales es que esta metodología de innovación educativa ayuda a trabajar la competencia comunicativa y el fomento de la responsabilidad y la autonomía del alumnado en el aprendizaje, aspectos que junto a otros favorecen un aprendizaje más efectivo. Todos estos aspectos se recomiendan en el DECRETO 242/2019, de 25 de octubre, de establecimiento de las enseñanzas y del currículum de idiomas de régimen especial en la Comunitat Valenciana.

La innovación propuesta surge de la necesidad de aprovechar el trabajo que realizan los alumnos durante la lectura del libro para que, gracias a las reuniones del club de lectura en el horario lectivo, les sirva como vehículo para trabajar la expresión oral a partir de reuniones en las que los alumnos trabajarán en grupo siguiendo unas directrices claras planteadas por la profesora.

Además de esto y relacionado con este concepto, se trabaja la oralidad tal como la define Núñez Delgado (2011): "la oralidad constituye una capacidad comunicativa que configura modos de percibir, de pensar y, por supuesto, de expresar el mundo". Esta idea de pensamiento crítico entronca con el trabajo de Finkel (2008), que utiliza el recurso del texto escrito para que aflore la reflexión en el proceso de aprendizaje.

Por todo ello, podemos aplicar a esta experiencia didáctica la afirmación de que "lo que realmente supone una innovación educativa es una suma sinérgica entre crear algo nuevo, el proceso en el que se aplica y la 
aportación de una mejora como resultado del proceso, y todo ello con una dependencia del contexto en el que se desarrolla y aplica la supuesta innovación" (García-Peñalvo, 2015).

Como respuesta a la detección de una falta de conciencia por parte de los alumnos de la importancia de la práctica de la oralidad y al expresión oral a partir de la lectura de una obra literaria, se ha diseñado un cuestionario cuya finalidad es conocer su percepción sobre este punto, así como su autoevaluación respecto a su rendimiento durante la actividad.

\section{Objetivos}

Con el estudio de caso que se presenta en esta comunicación se pretende:

1. Conocer la percepción del alumnado con respecto a:

1. su experiencia general en el club de lectura;

2. su rendimiento en la comprensión lectora;

3. su rendimiento en la expresión oral.

2. Fomentar la reflexión y la autoevaluación crítica sobre su trabajo de lectura individual en casa y su participación en las sesiones compartidas de práctica de la oralidad en el aula.

3. Hacer reflexionar al alumno sobre las mejoras en su trabajo para futuros clubs de lectura para sacarles el máximo rendimiento.

4. Concienciar al alumno sobre los aspectos que han mejorado más dentro de su competencia en expresión oral gracias a la participación en el club de lectura.

\section{Desarrollo de la innovación}

La metodología que se ha seguido en esta innovación didáctica en el aula ha consistido en primer lugar la elección del libro que los estudiantes van a leer. Los alumnos realizan dos clubs de lectura durante el curso en los cuales leen dos libros diferentes. En el caso del primero, todo el grupo de alumnos lee el mismo libro que ha seleccionado la profesora. El objetivo es que conozcan la actividad y adquieran las competencias necesarias para poder trabajar de forma más autónoma durante el segundo club de lectura. En este caso, son los propios alumnos, agrupados en grupos pequeños en función de sus gustos literarios, eligen el libro que van a leer. Cada grupo, por tanto, puede estar realizando una lectura diferente.

El paso siguiente es que en gran grupo o en grupos pequeños, en función de la distribución del alumnado comentada anteriormente, éstos dividen sus libros en cuatro partes, una por cada sesión del club de lectura establecida por la profesora y que tiene un carácter quincenal. Antes de cada una de las fechas del calendario de reuniones los alumnos tienen que leer en casa individualmente en casa la parte consensuada previamente.

Durante el proceso de lectura se les pide a los alumnos que realicen las siguientes tareas:

- Completar un punto de libro de elaboración propia siguiendo la propuesta de la profesora para que anoten el léxico más relevante que consideran interesante. El objetivo es que lo tengan a mano y lo utilicen durante la sesión de práctica oral con sus compañeros en clase. 
- Elegir un párrafo o parte relevante que les parezca interesante durante la lectura para compartirlo mediante la lectura en voz alta en las reuniones del club de lectura.

- Anotar cualquier duda o comentario sobre los capítulos correspondientes a la sesión para compartirlos y, en su caso, resolverlos durante la reunión.

Posteriormente, los alumnos se agrupan en cada una de las sesiones en grupos de máximo cuatro personas, en el caso en el que toda la clase lee el mismo libro, o en los grupos establecidos según los gustos literarios que se habían establecido previamente.

Durante las sesiones, que tenían una duración de media hora como mínimo, los alumnos comentan en inglés los párrafos que han elegido, el léxico y las preguntas, dudas o comentarios que han preparado previamente en casa.

Además, para fomentar el aprendizaje dialógico, los alumnos tienen asignados diferentes roles que se van turnando en cada reunión:

- controlador de tiempo, que está pendiente de que tratan todos los puntos en función del tiempo de duración determinado por la profesora para la reunión,

- redactor, que se encarga de tomar notas de las preguntas sugeridas por el grupo para los alumnos que no han asistido a clase y se han perdido esa sesión del club de lectura,

- recopilador de léxico, que controla el vocabulario y las expresiones que se utilizan durante la reunión y que los miembros del grupo han seleccionado del libro,

- coordinador, que supervisa la participación de todos los miembros del grupo, se asegura de que todos practican la expresión oral y comparten con el resto de compañeros el trabajo que han hecho individualmente en casa.

Cabe destacar que, tal como se ha mencionado al principio de esta comunicación, además de la oralidad la innovación metodológica de los clubs de lectura en el aula ayuda a desarrollar competencias transversales. Una de ellas es el sentido crítico, que se trabaja mediante el análisis del contexto de la obra literaria y su comparación con la realidad social. Al mismo tiempo también se desarrolla el pensamiento reflexivo, puesto que la lectura nunca deja indiferente al lector. Asimismo, esta práctica supone una mejora para la docencia porque es un recurso didáctico que genera diálogo y reflexión por parte del alumnado, mucho más que si se siguiera el libro de texto redactado por una editorial.

\section{Resultados}

Al finalizar la actividad, se les administró a los alumnos de los cursos 2018/2019 y 2019/2020 un cuestionario elaborado con un formulario de Google para evaluar la actividad. En este apartado se muestran los resultados del cuestionario completado por los alumnos según los objetivos establecidos más arriba.

El cuestionario incluye algunas preguntas sobre el club de lectura en general y otras destinadas a fomentar la autoevaluación del alumnado con respecto a su compromiso con su propio proceso de aprendizaje mediante esta metodología. Algunas de las preguntas se han planteado siguiendo la escala Likert (de 1 a 5 , siendo 5 el valor más alto) y en algunos casos se ha preferido dejarlas como respuesta abierta para conocer la opinión del alumnado y fomentar el pensamiento crítico y la práctica reflexiva. 
En primer lugar, vemos el porcentaje de alumnos que han respondido al cuestionario según los diferentes curso y niveles en los que están participando en el club de lectura. En total han respondido al cuestionario 38 alumnos, según se puede ver en la siguiente figura 1.

\section{Curso en el que has realizado el club de lectura.}

38 respostes

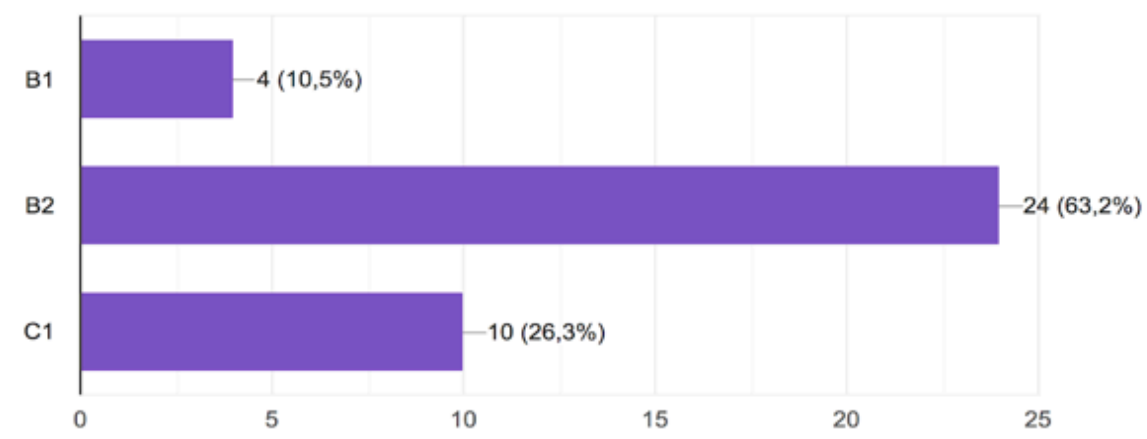

Fig. 1 Porcentaje de alumnos que han participado en el cuestionario según sus niveles de conocimiento de la lengua

A continuación se presentan los resultados relacionados con los objetivos planteados más arriba en el apartado 2 .

\section{Objetivo 1.1: Conocer la percepción del alumnado con respecto su experiencia general en el club de lectura.}

Se observa que casi la totalidad de los alumnos que han respondido al cuestionario están muy satisfechos con esta metodología, ya que un 50\% de ellos le dan una puntuación de 5, la máxima establecida, y un $39,5 \%$ la califican con un 4 . Solo un $4 \%$ (4 alumnos) la puntúan con un 3 y ninguno con menos de este valor. Estos datos muestran que les ha gustado trabajar la lectura de libros por medio de los clubs de lectura. Ver figura 2.

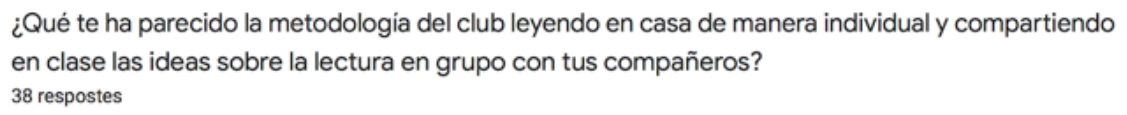

20

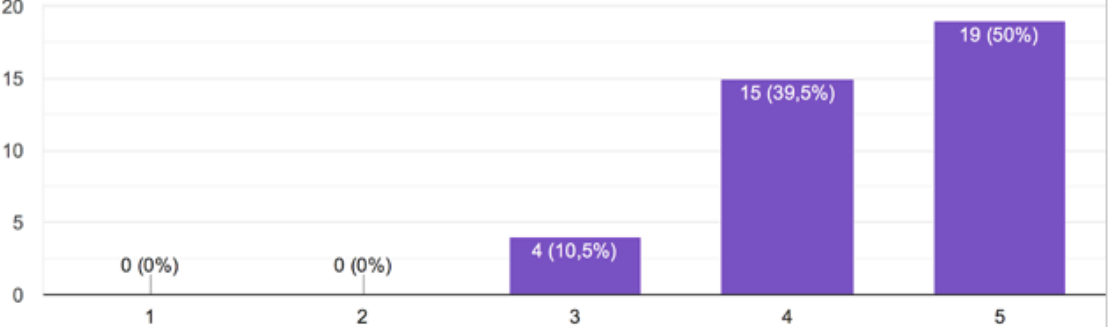

Fig. 2 Valoración de la experiencia

(c) DYY-NC-ND 2020, Universitat Politècnica de València 


\section{Objetivo 1.2: Conocer la percepción del alumnado con respecto a su rendimiento en la comprensión lectora.}

Como en el caso anterior, la amplia mayoría de los alumnos consideran que esta actividad les ha ayudado a mejorar su comprensión lectora, en concreto un $84,2 \%$ si tomamos los dos valores de puntuación más altos ( 4 y 5 ). En este caso se mantiene un $10 \%$ que puntúa un 3 y sí hay dos participantes, que corresponden al 5,3\%, que no consideran que los clubs de lectura hayan mejorado esta competencia. Ver figura 3.

\section{¿Crees que participar en el club de lectura te ha ayudado a mejorar tu comprensión lectora? 38 respostes}

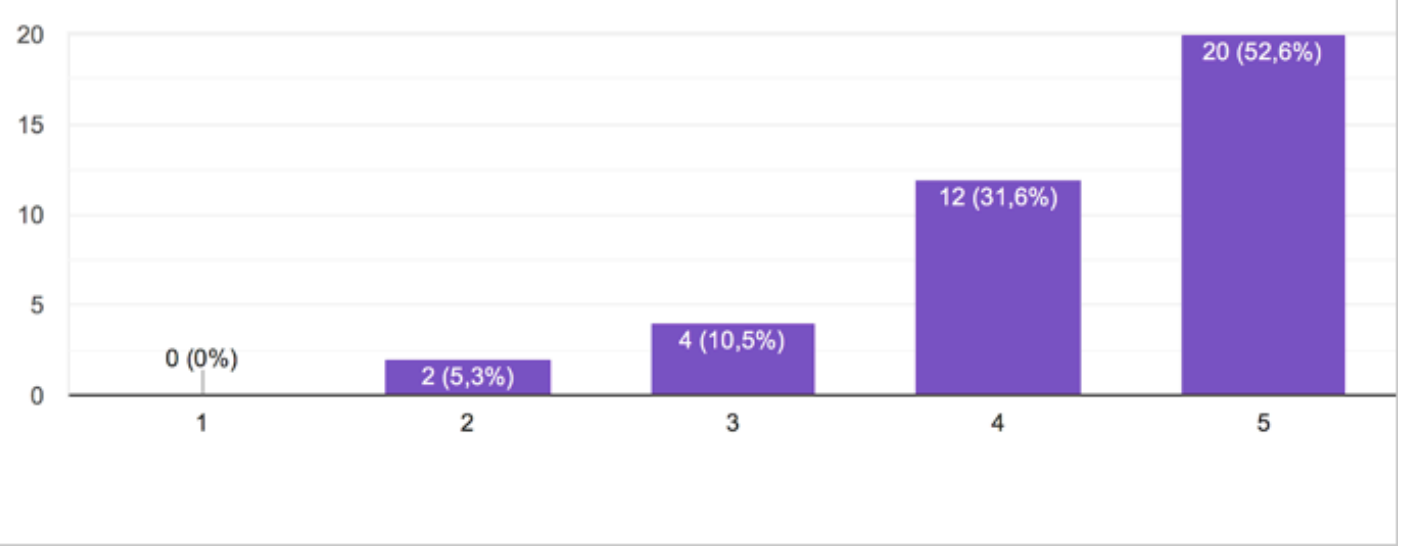

Fig. 3 Percepción con respecto al rendimiento en la comprensión lectora

\section{Objetivo 1.3: Conocer la percepción del alumnado con respecto a su rendimiento en la expresión oral.}

También en este caso los participantes consideran que participar en el club de lectura les ha ayudado a mejorar la competencia oral, con 30 de ellos que puntúan entre 4 y 5, mientras que 8 alumnos no parecen estar tan satisfechos con este apartado como con el anterior.

Cabe destacar que ninguno de los participantes ha valorado esta actividad con la puntuación mínima, lo cual quiere decir que sí son conscientes de su utilidad, si bien en cada caso le otorgan mayor o menor importancia. Esto puede deberse a que como se trata de un club de lectura, no valoran debidamente el apartado de la expresión oral. Ver figura 4. 


\section{¿Crees que participar en el club de lectura te ha ayudado a mejorar tu expresión oral?} 38 respostes

20

15

$17(44,7 \%)$

10

0

5 5

0

0

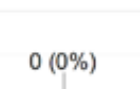

$3(7,9 \%)$

2

3

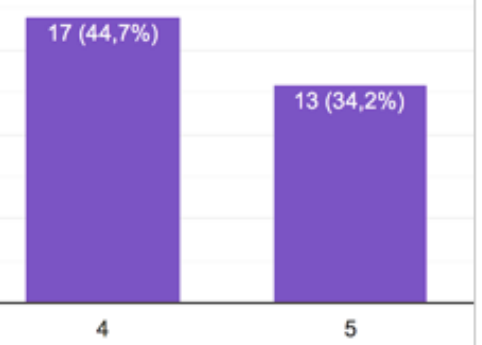

Fig. 4 Valoración de su experiencia para el desarrollo de la expresión oral

\section{Objetivo 2: Fomentar la reflexión y la autoevaluación crítica sobre su trabajo de lectura individual en casa y su participación en las sesiones compartidas de práctica oral en el aula.}

Las dos preguntas siguientes referidas a este objetivo se han planteado para que los alumnos autoevalúen su desempeño durante la actividad, tanto en la parte de trabajo individual realizada en casa, como en las sesiones grupales que se han llevado a cabo en el aula.

En ambos casos la puntuación que se otorgan es bastante alta, ya que tanto en uno como en el otro más de la mitad valoran su trabajo sobre 4 o 5 . Esto demuestra que están satisfechos o muy satisfechos con el trabajo que han realizado.

No obstante esta similitud, llama la atención que la puntuación de 5 en ambas gráficas es exactamente la misma y la diferencia está en los valores de 3 y 4 . En general, los alumnos están más satisfechos con el trabajo que han realizado en el aula en grupo que con el que han llevado a cabo en su casa de forma individual. Ver figuras 5 y 6 . 
¿Cómo evaluarias tu proceso de lectura individual en casa? (Organización del tiempo, ritmo de lectura, búsqueda de léxico nuevo, lectura en voz alta, etc.)

38 respostes

15

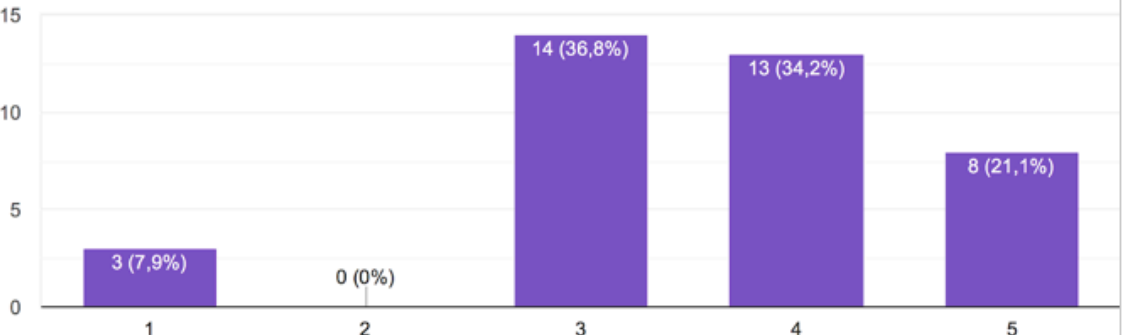

3

4

Fig. 5 Valoración del proceso de lectura en casa

¿Cómo evaluarias tu participación en las sesiones grupales de práctica oral en el aula? (Participación en la conversación, aporte de ideas, etc.)

38 respostes

20

15

$17(44,7 \%)$

10

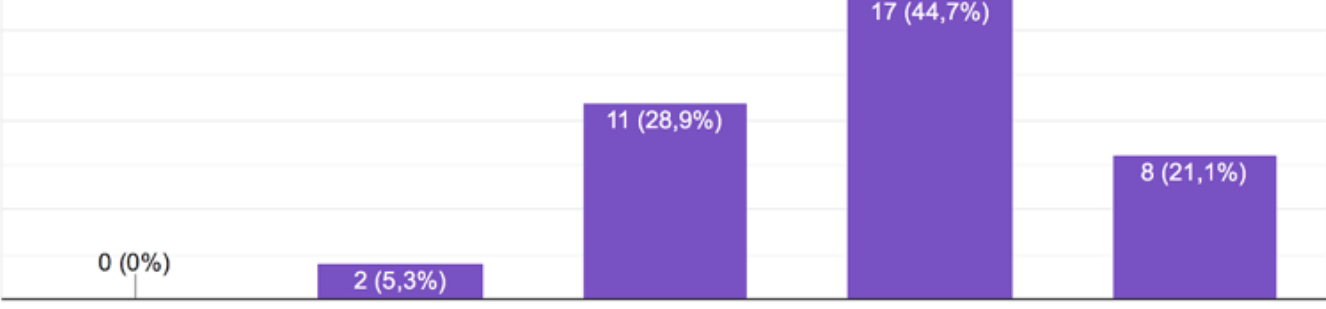

0

2

3

4

5

Fig. 6 Valoración del proceso de lectura en el aula

\section{Objetivo 3: Hacer reflexionar al alumno sobre las mejoras en su trabajo durante futuros clubs de lectura para sacarles el máximo rendimiento.}

En este caso la pregunta era de respuesta abierta y, a pesar de que las cuestiones estaban planteadas en castellano, en la tabla 1 se puede observar que algunos alumnos han utilizado el inglés para responder. Seguramente esto es debido a que los alumnos están aprendiendo este idioma y, por tanto, es la lengua de comunicación en todo momento en el aula.

A continuación se detallan las respuestas de los alumnos agrupadas según las propuestas de mejoran que se plantean para futuros clubs de lectura. 
Tabla 1 Propuestas de mejoras de los alumnos

\begin{tabular}{|c|c|}
\hline \multirow{3}{*}{$\begin{array}{l}\text { a) Participación } \\
\text { en las sesiones grupales. }\end{array}$} & $\begin{array}{l}\text { - Intentaría hablar más. } \\
\text { - Hablar más. Pensar menos. } \\
\text { - Mayor participación. } \\
\text { - Participar más activamente. } \\
\text { - Aportar más ideas. }\end{array}$ \\
\hline & $\begin{array}{l}\text { - Haría preguntas entre los miembros del club para una óptima } \\
\text { comprensión. Y al preguntar se practica la expresión oral } \\
\text { mejorando la pronunciación y la fluidez. } \\
\text { - No comentaría sólo un resumen del capítulo, sino analizaría también } \\
\text { si el autor ha usado dobles significados, o cuál era su intención al } \\
\text { escribirlo, cosas así. }\end{array}$ \\
\hline & $\begin{array}{l}\text { - Nothing. I was able to summarize the plot with my classmates and we } \\
\text { talked about new words and expressions we found. (Nada. Fui } \\
\text { capaz de resumir el argumento con mis compañeros y hablamos } \\
\text { sobre palabras nuevas y expresiones que encontramos.) }\end{array}$ \\
\hline \multirow[t]{2}{*}{$\begin{array}{l}\text { b) Preparación previa a la } \\
\text { reunión del club de lectura. }\end{array}$} & $\begin{array}{l}\text { - Leer más para poder participar de forma más activa. } \\
\text { - Leer más el libro previamente. } \\
\text { - Mayor preparación en casa para que en clase tenga más recursos. } \\
\text { - Mi preparación previa, ya que mi ritmo de lectura ha sido más lento } \\
\text { que el del resto de compañer@s. }\end{array}$ \\
\hline & $\begin{array}{l}\text { - Llevar alguna idea pensada previamente sobre temas de los que } \\
\text { hablar. } \\
\text { - Llevar un listado del vocabulario, había llevado el significado } \\
\text { apuntado en el mismo libro. } \\
\text { - Preparar preguntas de las cosas del libro que no haya entendido. }\end{array}$ \\
\hline \multirow{4}{*}{$\begin{array}{l}\text { c)Aspectos relacionados con la } \\
\text { expresión oral. }\end{array}$} & $\begin{array}{l}\text { - Me obligaría a hablar más, para así practicar más el oral. } \\
\text { - Mejorar la fluidez a la hora de hablar y el vocabulario. }\end{array}$ \\
\hline & $\begin{array}{l}\text { - I need to improve my oral fluency. (Tengo que mejorar mi fluidez } \\
\text { oral.) } \\
\text { - I need to improve my speaking fluency. (Tengo que mejorar mi fluidez } \\
\text { a la hora de hablar.) }\end{array}$ \\
\hline & - Tengo que mejorar la pronunciación. \\
\hline & - More interaction from me. (Mayor interacción por mi parte.) \\
\hline \multirow{4}{*}{ d) Trabajo cooperativo } & $\begin{array}{l}\text { - Tomar notas con los aspectos que me gustaría tratar, para resolver mis } \\
\text { posibles dudas de manera grupal. }\end{array}$ \\
\hline & - Ayudar más a mis compañeros. \\
\hline & $\begin{array}{l}\text { - Hablar más y mostrar mis experiencias en lugar de escuchar solo las } \\
\text { de los demás. }\end{array}$ \\
\hline & $\begin{array}{l}\text { - Intentaría que todos pudieran participar de algún modo. Hacer más } \\
\text { participativa esta actividad para aquellos que por alguna razón u } \\
\text { otra no han podido terminar el libro, pero puedan dar su punto de } \\
\text { vista sobre el mismo, y así no recaiga toda la responsabilidad a la } \\
\text { hora de debatir en quienes han leído el libro entero. } \\
\text { - Participación más equilibrada: los más tímidos sólo escuchan, los más } \\
\text { lanzados copan un porcentaje muy elevado del tiempo. }\end{array}$ \\
\hline
\end{tabular}


e) Otros.

Cabe destacar que algunas de las propuestas de mejora coinciden con instrucciones que se han dado a los alumnos antes de realizar la actividad. Esto demuestra que no las habían seguido y mediante la autoevaluación se han dado cuenta de que para aprovechar al máximo la actividad en el futuro tendrán que hacerlo.

\section{Objetivo 4: Concienciar al alumno sobre los aspectos que han mejorado más dentro de su competencia en expresión oral gracias a la participación en el club de lectura.}

Prácticamente todos los alumnos que han respondido al cuestionario consideran que esta actividad ha contribuido a mejorar su expresión oral, con un porcentaje de un 97,4\%. Ver figura 7.

\section{¿Crees que la participación en el club de lectura ha contribuido a mejorar tu expresión oral?}

38 respostes

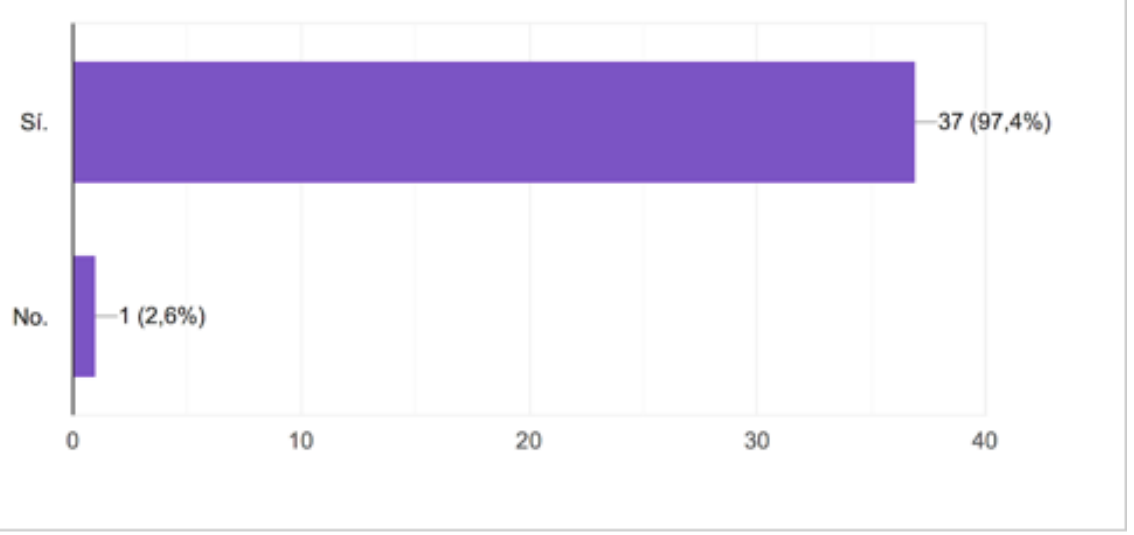

Fig. 7 Valoración general del club de lectura

A la pregunta sobre cómo creen que la participación en el club de lectura ha contribuido más en la mejora de su expresión oral, el $42 \%$ del alumnado considera que participar en el club de lectura les ha dado más confianza a la hora de expresarse en inglés, lo cual está directamente relacionado con la percepción que tienen ahora sobre mostrar mayor fluidez a la hora de expresarse en esta lengua $(23,7 \%)$. Estos datos suponen la consecución de uno los objetivos planteados al diseñar esta actividad y ponerla en práctica en el aula.

Otro de los objetivos de aprendizaje planteados para el club de lectura es la adquisición de léxico nuevo en contexto. Un $23,7 \%$ de los participantes en el cuestionario afirman que durante las sesiones grupales han utilizado palabras y expresiones aprendidas durante la lectura. Con este porcentaje se puede considerar que este objetivo se ha conseguido en buena medida. 
Por último, un 10,5\% consideran que también han adquirido mayor corrección a la hora de expresarse en inglés, es decir, que la práctica de compresión lectora y expresión oral les ha permitido tener menos errores gramaticales y de léxico. Ver figura 8.

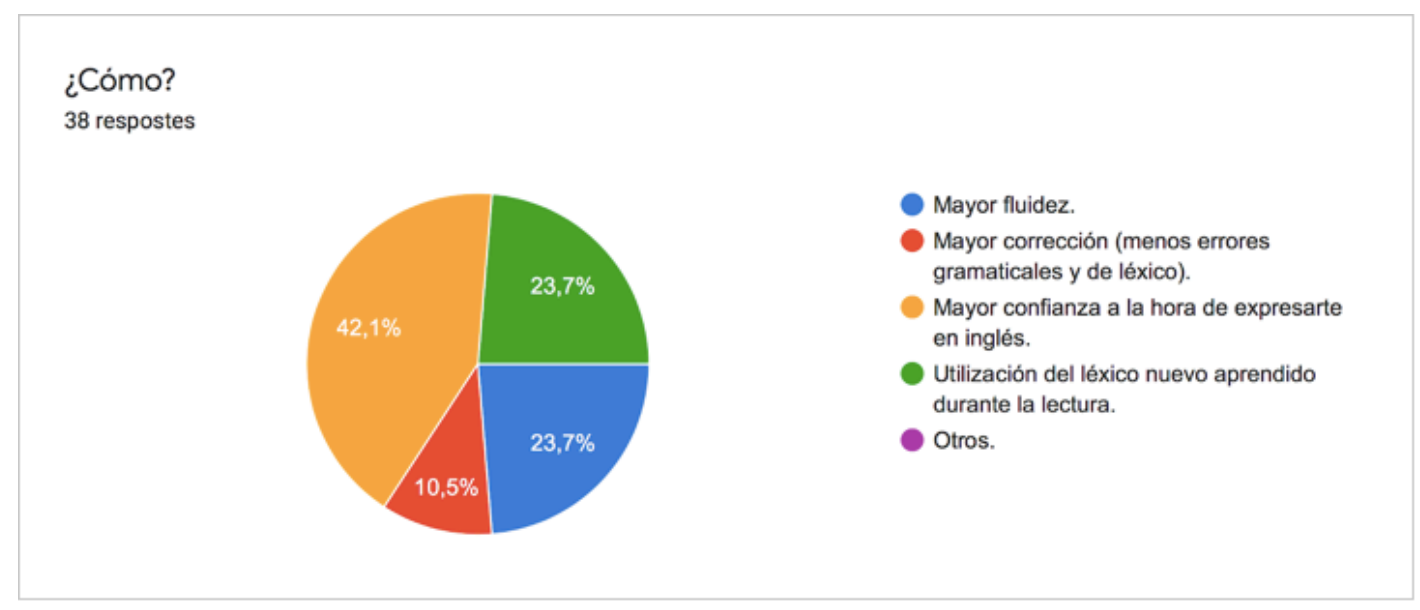

Fig. 8 Mejoras percibidas por los alumnos en su expresión oral durante las sesiones grupales en el aula

Tras analizar los resultados del cuestionario, se corrobora que la utilización de los clubs de lectura en el aula de lengua demuestra ser una metodología bien valorada por los alumnos para desarrollar la comprensión lectora, algo que corrobora los resultados mencionados anteriormente sobre esta práctica didáctica.

En cuanto a la práctica de la oralidad, se ha demostrado que los alumnos también consideran que su expresión oral ha mejorado y plantean propuestas que se tendrán en cuenta a la hora de diseñar la actividad en próximos cursos.

\section{Conclusiones}

Según los resultados analizados en la presente comunicación, se demuestra que la innovación metodológica presentada sobre la utilización de los clubs de lectura en el aula de lengua cumple con las características citadas por García-Peñalvo (2015) acerca de la realización de cambios metodológicos que produzcan mejoras en los resultados de aprendizaje. Asimismo, se demuestra que esta actividad no es sólo útil para el desarrollo de la competencia lectora de los alumnos, como cabría suponer, sino que también garantiza la mejora de su expresión oral y el pensamiento y la reflexión crítica a través de la práctica de la oralidad en el aula.

En relación a los objetivos planteados, se puede afirmar que se han cumplido de manera satisfactoria y están en consonancia con el curriculum vigente en la Comunidad Valenciana. El cumplimiento de estos objetivos hace que se esté en consonancia con las directrices de la actual legislación en materia de enseñanza de lenguas extrajeras en Escuelas Oficiales de Idiomas y, por ende, con el Marco Común Europeo de Referencia para las Lenguas, en el que se basa dicha legislación. 
Para futuros clubs de lectura, y en función de la autoevaluación y reflexión crítica que los alumnos han hecho sobre sí mismos a través de los comentarios de los cuestionarios, cabe destacar la necesidad de hacer hincapié en el aula de la importancia que tiene la correcta realización del proceso de lectura individual en casa y su participación activa en las sesiones de práctica oral en el aula. Esto ayudará a que se den cuenta de los objetivos de la actividad y sean conscientes de que los clubs de lectura no solo les ayudan a mejorar su comprensión lectora, sino que también son útiles para mejorar su expresión oral. Esta mejora se llevará a cabo mediante la creación de fichas de trabajo y material adicional.

Por último, dada la satisfacción del alumnado con respecto a esta innovación metodológica en el aula de lengua, se considera que la utilización de esta actividad por parte del profesorado es transferible a otras lenguas dentro de las enseñanzas de Escuelas Oficiales de Idiomas. También se recomienda su utilización en otros niveles educativos, siempre haciendo las adaptaciones pertinentes en función del contexto docente y características del alumnado, así como de la legislación y el curriculum vigente en cada una de ellas.

\section{Referencias}

ÁLVAREZ ÁLVAREZ, C. (2016). “Clubs de lectura ¿Una práctica relevante hoy?” en Información, cultura y sociedad/35, 91-106. ISSN:1851-1740.

$<$ http://repositorio.filo.uba.ar/handle/filodigital/11201> [Consulta: 15 de marzo de 2020]

FINKEL, D. Traducción Barberá, O. (2008). Dar clases con la boca cerrada. Universidad de Valencia. España.

GARCÍA-PEÑALVO, F. J. (2015). "Mapa de tendencias en Innovación Educativa" en Education in the Knowledge Society, 16(4),6-23. e-ISSN: 2444-8729.

<https://revistas.usal.es/index.php/eks/article/view/eks2015164623/13764> [Consulta: 11 de Marzo de 2020]

KOUTSOMPOU, V. (2015). "The Use of Literature in the Language Classroom: Methods and Aims" en International Journal of Information and Education Technology, Vol. 5, No.1, January 2015, 74-79.

$<$ http://www.ijiet.org/papers/479-H10011.pdf> [Consulta: 18 de marzo de 2020]

MOLINER GARCÍA, O., SANHAUJA RIBÉS, A., \& BENET-GIL, A. (2017). Prácticas inclusivas en el aula desde la investigación-acción.

<http://repositori.uji.es/xmlui/handle/10234/168902> [Consulta: 15 de marzo de 2020]

NÚÑEZ DELGADO, M. P. (2011). "Espejos y ventanas: Dimensiones de la oralidad en el ámbito educativo" en Enunciación, 16(1), 136-150.

<https://doi.org/10.14483/22486798.3594> [Consulta: 15 de marzo de 2020] 